\title{
Muon reconstruction performance of the ATLAS detector in 2016
}

\author{
Luigi Marchese ${ }^{1, a}$ on behalf of the ATLAS Collaboration \\ ${ }^{1}$ University of Oxford
}

\begin{abstract}
Muons are of key importance to study some of the most interesting physics topics at the LHC. We show the status of the performance of the muon reconstruction in the analysis of proton-proton collisions at the LHC, recorded by the ATLAS detector in 2016. Reconstruction efficiency and momentum resolution have been measured using $J / \psi$ and $Z$ decays for different classes of reconstructed muons.
\end{abstract}

\section{Introduction}

Muon reconstruction performance of the ATLAS detector during the LHC run at $\sqrt{s}=7-8 \mathrm{TeV}$ have been documented in [1]. In 2013-2015 during the shutdown preceding the LHC Run 2, the LHC was upgraded to increase the center-of-mass energy from 8 to $13 \mathrm{TeV}$. Additional muon chambers and a new innermost Pixel layer, the B-Layer, have been added to the ATLAS detector, providing measurements closer to the interaction point. Moreover, the muon reconstruction software was updated and improved. A first result of the muon reconstruction performance of the ATLAS detector in 2015 at $\sqrt{s}$ $=13 \mathrm{TeV}$ can be found in [2]. In this note we describe the performance of the muon reconstruction in the dataset collected at $\sqrt{s}=13 \mathrm{TeV}$ in 2016, corresponding to an integrated luminosity of $33.3 \mathrm{f} b^{-1}$. We focus on the muon $p_{T}$ range from $\sim 6 \mathrm{GeV}$ to $\sim 100 \mathrm{GeV}$, in which large samples of calibration data are available from $J / \psi \rightarrow \mu \mu$ and $Z \rightarrow \mu \mu$. Reconstruction efficiency, transverse momentum resolution and momentum scales are measured in the various regions of the detector and for different muon momenta. These results are compared to corrected Monte Carlo (MC) simulations showing a good agreement.

This note is structured as follows: Section 2 decribes the ATLAS subdetectors that are most relevant to this study; Section 3 describes the muon reconstruction and identification in ATLAS during the LHC Run 2; Section 4 and Section 5 describe the muon reconstruction efficiency and the muon momentum scale and resolution. Finally, in Section 6 we present the final conclusions.

\section{ATLAS detector}

An accurate description of the ATLAS detector can be found in [3, 4]. Muons are initially identified and reconstructed by combining information from the inner detector (ID) and the muon spectrometer (MS), supplemented by information from the calorimeters.

The ID is the main tracker providing accurate measurements of the muon track parameters inside an

ae-mail: luigi.marchese@cern.ch 
axial magnetic field of $2 \mathrm{~T}$. It consists of three subdetectors: the silicon pixels (Pixels) and the semiconductor tracker (SCT) with a pseudorapidity coverage up to $|\eta|=2.5$, and the transition radiation tracker (TRT) with a pseudorapidity coverage up to $|\eta|=2.0$.

The MS is the outermost ATLAS detector. It operates in the pseudorapidity region $|\eta|=2.7$. It is designed to provide momentum measurements with a relative resolution better than $3 \%$ over a wide $p_{T}$ range and up to $10 \%$ at $\sim 1 \mathrm{TeV}$. It consists of one barrel $(|\eta|<1.05)$ and two endcap sections $(1.05$ $<|\eta|<2.7)$. A system of three large superconducting air-core toroidal magnets provides a magnetic field with a bending integral of about $2.5 \mathrm{Tm}$ in the barrel and up to $6 \mathrm{Tm}$ in the endcaps. Three layers of resistive plate chambers (RPCs) $(|\eta|<1.05)$ and three layers of thin gap chambers (TGC) $(1.05<\eta<2.4)$ serve as trigger chambers for the detector and provide $(\eta, \phi)$ position measurements with typical spatial resolution of 5-10 mm. Three layers of monitored drift tube (MDTs) chambers $(|\eta|$ $<2.7$ ) provide precise muon momentum measurements with each chamber providing six to eight $\eta$ measurements along the muon trajectory. In the pseudorapidity region $|\eta|>2$ a quadruplet of cathode strip chambers (CSC) replaces the innermost layer of MDTs.

During the shutdown preceding the LHC Run 2, the MS was completed to its initial design specifications [4] by adding the last missing chambers in the transition region between the barrel and the endcaps $(1.0<\eta<1.4)$. In addition, four RPC-equipped MDT chambers were installed inside the two elevator shafts to improve the acceptance in that region relative to Run 1.

\section{Muon reconstruction and identification}

Muons are initially reconstructed independently in the ID and MS. The information from individual subdetectors is then combined to form the muon tracks used in physics analyses. Muons are reconstructed like any other charged particles in the ID. In the MS, muons are reconstructed searching for hit patterns inside each muon chamber to form segments. Tracks candidate are then built by performing a general fit of all the segments in different layers. A track candidate is considered only if the fit satisfies the selection criteria. Four muon types are defined depending on which subdetectors are used in the reconstruction. About $96 \%$ of muons are recontructed by performing a global refit that uses hits from both the ID and MS. This defines the combined $(C B)$ type. The other types correspond to tagged ID tracks with muon signatures in the MS or the calorimeter.

Quality requirements are applied to muon types in order to suppress background, mainly originating from pion and kaon decays. High efficiency and a robust momentum measurement is guaranteed for prompt muons. Four muon identification selections (Medium, Loose, Tight and High- $p_{T}$ ) are available to meet the different requirements of the various physics analyses. The Medium category provides the default selection for muons in ATLAS. It is designed to minimize the systematic uncertainty associated with muon reconstruction and momentum calibration. The Loose identification criteria maximise the reconstruction efficiency providing good-quality tracks. This category is specifically included for reconstructing Higgs boson candidates in the four-lepton final state. Tight muons are selected to maximize the purity of muons, though sacrifing the muon reconstruction efficiency. Finally, the High- $p_{T}$ selection criteria aims to maximize the momentum resolution for tracks with transverse momentum above $100 \mathrm{GeV}$. This category is used specifically for searches for new high-mass resonances.

\section{Reconstruction efficiency}

Since muons are reconstructed independently in the ID and MS sub-detectors, the reconstruction efficiency can be evaluated by identifying a muon in one of the two sub-systems, check how many times 
it is matched to a muon candidate in the other system, and then combine the two efficiencies. The tag-and-probe method, described in [1], is used to measure the muon reconstruction and isolation efficiency. The method is based on the selection of an almost pure muon sample from $J / \psi \rightarrow \mu \mu$ or $Z \rightarrow \mu \mu$ events. One leg of the decay (tag) is identified as a Medium muon that fires the trigger and the second leg (probe) is reconstructed by a system independent of the one being studied. The $J / \psi$ sample covers the low transverse momentum range $4.5<p_{T}<15 \mathrm{GeV}$, while the $Z$ sample extends the measurement up to $110 \mathrm{GeV}$. A probe is considered successfully reconstructed if a selected muon is found within a cone in the $(\eta, \phi)$ plane of size $\left.\Delta R=\sqrt{(} \Delta \eta^{2}+\Delta \phi^{2}\right)<0.05$ around the probe track. $Z$ events are selected by requiring muon pairs with an invariant mass within $10 \mathrm{GeV}$ of the $\mathrm{Z}$ boson mass and a transverse momentum of at least $10 \mathrm{GeV}$ for the probe muon, while the tag muon is required to satisfy the Medium muon identification selections. $J / \psi$ tag-probe pairs are selected within the invariant mass window of 2.7-3.5 GeV and requiring a transverse momentum of at least $5 \mathrm{GeV}$ for each muon. The tag muon is required to be identified as a Medium and to have triggered the readout of the event.

A small contribution of about $0.1 \%$ of the selected tag-probe pairs comes from sources other than $Z \rightarrow \mu \mu$ events. These background sources are estimated using MC simulation and data-driven techniques. They are finally subtracted. The background contamination for the $J / \psi$ events is estimated by performing a simultaneous maximum-likelihood fit of two statistically independent distributions of the invariant mass: events in which the probe is or is not successfully matched to the selected muon. Muon reconstruction efficiencies are extracted separately for MC and data with the same methods. Results are reported as a function of pseudorapidity and probe transverse momentum. The level of agreement of the measured reconstruction efficiency between data and MC is expressed as the ratio of these numbers, defining the "efficiency Scale Factor (SF)":

$$
S F=\frac{\epsilon^{\text {Data }}}{\epsilon^{M C}}
$$

This quantity describes the deviation of the simulation from data and is particularly relevant for physics analyses which use MC simulations that need to be corrected.

Figure 1 shows the muon reconstruction efficiency as a function of $\eta$ for $Z$ events in both MC and data for Medium, Tight and Loose muons. Muon reconstruction efficiencies are measured to be close to $99 \%$ for Medium and Loose muons and above $95 \%$ for Tight muons with all efficiencies in very good agreement with MC predictions. At $(\eta, \phi) \sim(-1.36,1.6)$ a clear inefficiency is due to a poorly aligned MDT chamber and is the main discrepancy between data and MC for Tight muons. Further local inefficiencies in the barrel region are also linked to temporary faults during data taking. The muon reconstruction efficiency as a function of $p_{T}$ is shown for Medium muons in Fig. 2. The plot displays the high reconstruction efficiency across a broad $p_{T}$ spectrum and the good agreement between data and MC.

\section{Muon momentum scale and resolution}

The ATLAS simulation contains an accurate description of the ATLAS detector. However, the level of detail is not enough to describe the muon momentum scale to the per mille level and the muon momentum resolution to the percent level needed by several physics analyses. A set of corrections is therefore applied to the simulated muon momentum. Corrections to the momentum resolution account for different physics effects, such as multiple scattering, local magnetic field distortions, energy loss due to fluctuations in the traversed material, intrinsic resolution effects, and residual misalignments of the detector. Only CB muons are used to extract the calibration parameters. More details can be 

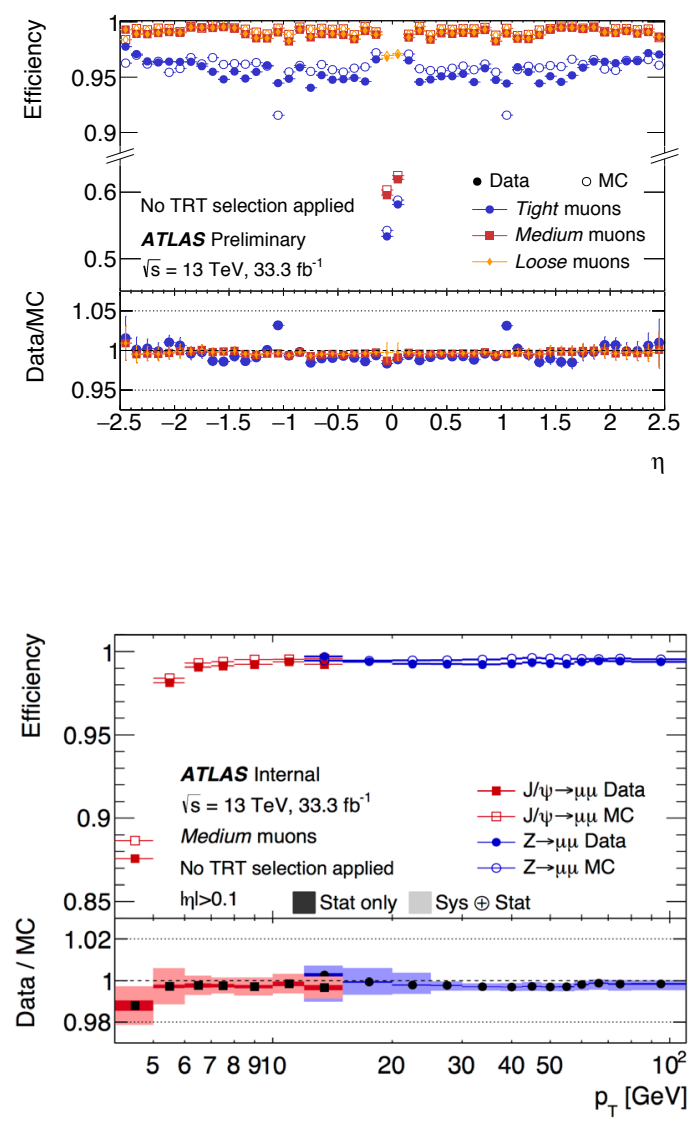

Figure 1. Muon reconstruction efficiencies for the Loose/Medium/Tight identification algorithms measured in $Z \rightarrow \mu \mu$ events as a function of the muon pseudorapidity for muons with $p_{T}>10 \mathrm{GeV}$. The prediction by the detector simulation is depicted as open circles, while filled dots indicate the observation in collision data with statistical errors. The bottom panel shows the ratio between expected and observed efficiencies, the efficiency scale factor. The errors in the bottom panel show the quadratic sum of statistical and systematic uncertainty. Figure from [5].

Figure 2. Muon reconstruction efficiencies for the Medium identification algorithm measured in $J / \psi \rightarrow \mu \mu$ and $Z \rightarrow \mu \mu$ events as a function of the muon momentum. The prediction by the detector simulation is depicted as empty circles (squares), while the full circles (squares) indicate the observation in collision data. Only statistical errors are shown in the top panel. The bottom panel reports the efficiency scale factors. The darker error bands indicate the statistical uncertainty, while the lighter bands indicate the sum of the statistical and systematic uncertainties in quadrature. Figure from $[5]$.

found in [1].

Certain systematic misalignment modes cause sagitta deviations, biasing the measured $p_{T}$ of positive and negative muons in opposite directions. Such effects are present in prompt data reconstruction and cause a small mass shift for charge-asymmetric final states hard to see on the $\mathrm{Z}$ mass. It also slightly increases the width of the $Z$ mass peak. These biases are studied and corrected in data by comparing the local inhomogeneities of the charge dependent dimuon mass to the mass of well-known neutral resonances. The correction improves the resolution of the dimuon invariant mass in Z-boson decays by $1 \%$ to $5 \%$, depending on $\eta$ and $\phi$. The systematic uncertainty associated to this correction is estimated for each muon using simulation. In Fig. 3 we show the effect of such a bias before (unfilled markers) and after (filled markers) the correction. After we correct positve and negative tracks show a better agreement. In Fig. 4 we show the residual effect of this bias in the $(\eta, \phi)$-map after we do correct. The residual biases are reduced to 0.2 per mille on the $\mathrm{Z}$ mass. In Fig. 5 and 6 we show the agreement between data and $\mathrm{MC}$ for the mass resolution and the dimuon mass scale of the pair which are directly related to the muon momentum resolution and scale. The dimuon mass resolution is obtained by fitting the width of the invariant mass peaks. The dimuon mass resolution is about $0.9 \%$ for $p_{T} \sim 6 \mathrm{GeV}$ in the barrel and $1.5 \%$ for $p_{T} \sim 45 \mathrm{GeV}$. The agreement between data and $\mathrm{MC}$ for the mean is within $0.1 \%$ in the barrel and $0.2 \%$ in the endcaps. 

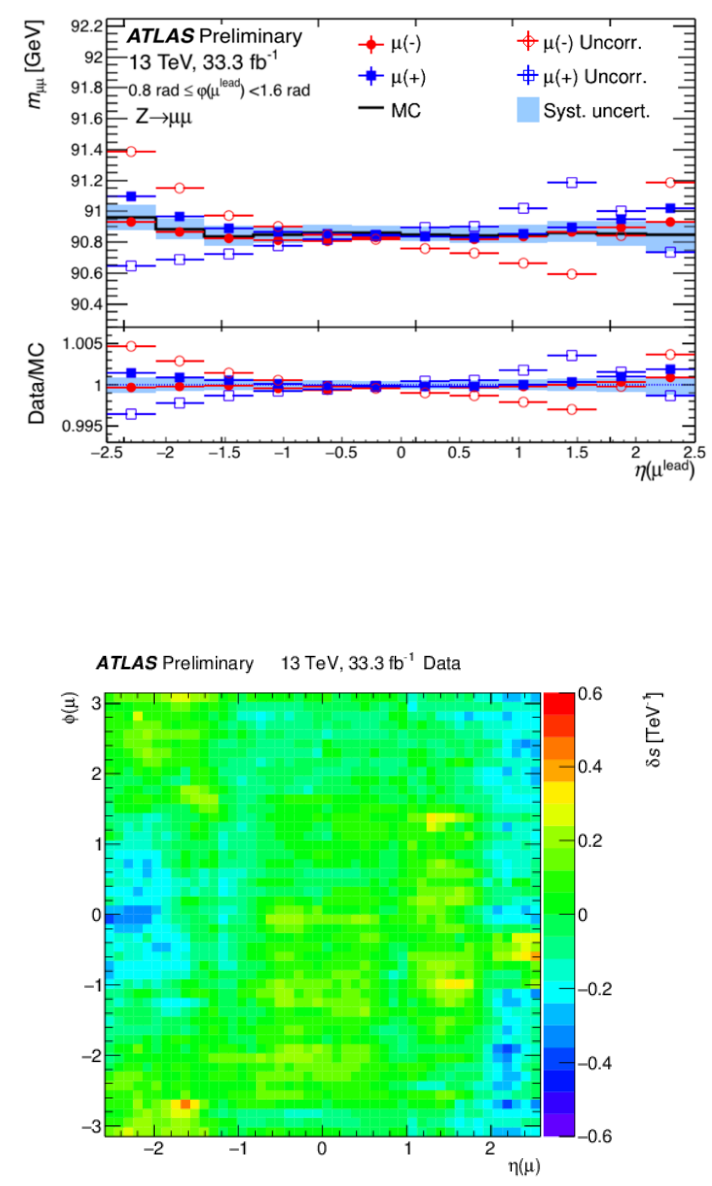

Figure 3. Transverse momentum $\left(p_{T}\right)$ scale as a function of pseudorapidity $(\eta)$ obtained from reconstructed $Z \rightarrow \mu^{+} \mu^{-}$candidates. The dataset, consisting of $33.3 \mathrm{fb}^{-1}$ of proton-proton collision data collected in 2016 by the ATLAS detector at a center-of-mass energy of $\sqrt{s}=13 \mathrm{TeV}$ is shown for reconstructed $Z \rightarrow \mu^{+} \mu^{-}$candidates with a positive leading muon (blue square markers) and negative leading muon (red circle markers) before (unfilled markers) and after (filled markers) correcting for possible misalignments of the Inner detector. Data are also compared to prompt $\mathrm{Z}$ simulation (continuous line). Systematic uncertainties (filled area) are derived from $\pm 1 \sigma$ variations of the smearing and scale parameters used to derive the correction. An uncertainty accounting for the missmodeling of the fitting function due to the missing background component is also taken into account. Figure from [5].

Figure 4. Residual local sagitta bias $\delta_{\mathrm{s}}$ for combined muons as a function of $\eta$ and $\varphi$, as evaluated from $Z \rightarrow \mu^{+} \mu^{-}$decays with transverse momenta from 5 $\mathrm{GeV}$ to $300 \mathrm{GeV}$, after application of the correction to data. Figure from [5].

\section{Conclusion}

The performance of the ATLAS muon reconstruction has been measured using $33.3 \mathrm{f} b^{-1}$ of data from pp collisions at $\sqrt{s}=13 \mathrm{TeV}$ recorded in 2016. $J / \psi$ and $Z$ decays allow for precise measurements of the muon reconstruction efficiency as well as of the muon momentum resolution and scale over a wide $p_{T}$ range. Results show a good agreement between data and the corrected MC simulation. 

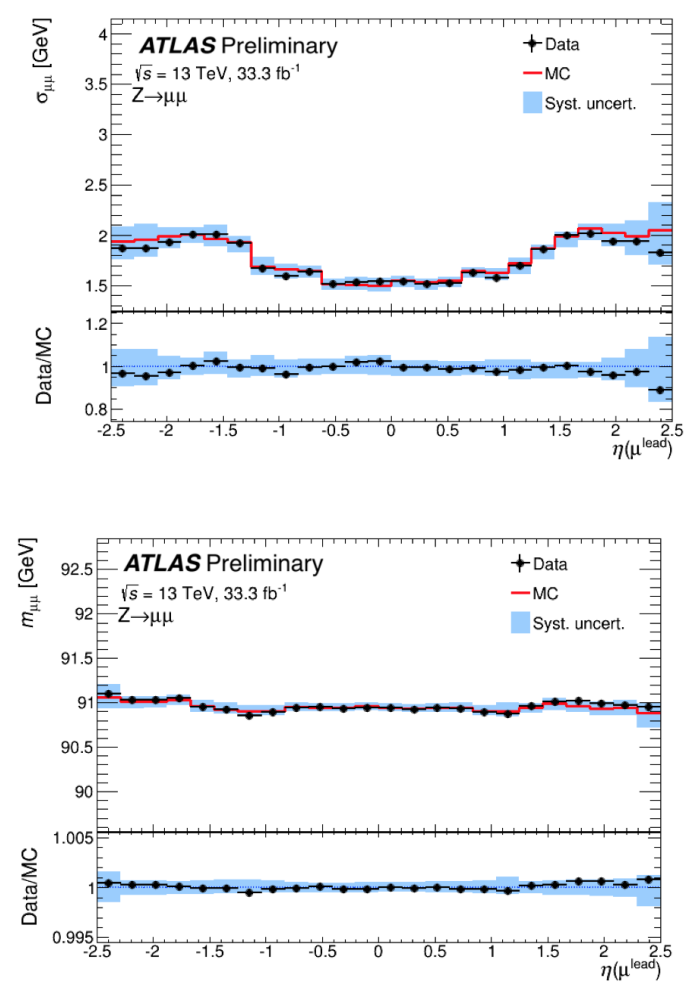

Figure 5. Transverse momentum $\left(p_{T}\right)$ resolution as a function of pseudorapidity $(\eta)$ obtained from reconstructed $Z \rightarrow \mu^{+} \mu^{-}$candidates. The dataset, consisting of $33.3 \mathrm{fb}^{-1}$ of proton-proton collision data collected in 2016 by the ATLAS detector at a centre-of-mass energy of $\sqrt{s}=13 \mathrm{TeV}$ (filled markers), is compared to prompt $Z$ simulation (continuous line). Muons are reconstructed combining Inner Detector and Muon Spectrometer tracks. Each event must contain two opposite-charge muons with $22 \mathrm{GeV}<p_{T}<300 \mathrm{GeV}$ and $|\eta|<2.5$. The binning is defined according to the $\eta$ of the leading muon. Figure from [5].

Figure 6. Transverse momentum $\left(p_{T}\right)$ scale as a function of pseudorapidity $(\eta)$ obtained from reconstructed $Z \rightarrow \mu^{+} \mu^{-}$candidates. The dataset, consisting of $33.3 \mathrm{fb}^{-1}$ of proton-proton collision data collected in 2016 by the ATLAS detector at a centre-of-mass energy of $\sqrt{s}=13 \mathrm{TeV}$ (filled markers), is compared to prompt $Z$ simulation (continuous line). Muons are reconstructed combining Inner Detector and Muon Spectrometer tracks. Each event must contain two opposite-charge muons with $22 \mathrm{GeV}<p_{T}<300 \mathrm{GeV}$ and $|\eta|<2.5$. Figure from [4]. The binning is defined according to the $\eta$ of the leading muon. Figure from [5].

\section{References}

[1] ATLAS Collaboration, Measurement of the muon reconstruction performance of the ATLAS detector using 2011 and 2012 LHC proton-proton collision data, Eur. Phys. J. C74.11 (2014) 3130, arXiv: 1407.3935 [hep-ex].

[2] ATLAS Collaboration, Muon reconstruction performance of the ATLAS detector in proton-proton collision data at $\sqrt{(} s)=13 \mathrm{TeV}$, Eur. Phys. J. C76:292 (2016), arXiv:1603.05598 [hep-ex].

[3] ATLAS Collaboration, The ATLAS Experiment at the CERN Large Hadron Collider, JINST 3 (2008) S08003.

[4] ATLAS Collaboration, ATLAS muon spectrometer: Technical design report, CERN-LHCC-9722, ATLAS-TDR-10 (1997).

[5] https://atlas.web.cern.ch/Atlas/GROUPS/PHYSICS/PLOTS/MUON-2017-001/index.html 